\title{
Study of heteropolyacids for the hydroxylation of Naphthalene to naphthol
}

\author{
PengjinYe, Wenya Wang\&LeiWang*
}

Shanghai Institution of Technology, Shanghai 201418, China

\begin{abstract}
We prepared five heteropolyacid catalyst by one-pot method, the reaction temperature was $65 \mathrm{oC}$, reaction time $8 \mathrm{~h}$, hydrogen peroxide $3 \mathrm{~mL}$, naphthalene $1000 \mathrm{mg}$, H4PMo11VO40 $100 \mathrm{mg}$, naphthalene conversion was $30.3 \%$,the selectivity of naphthol was $46.7 \%$. The heteropoly acids have Keggin structure, but also have some hydroxylations catalytic activity in reaction of naphthalene to naphthol. Among which,the H4PMo11VO40 was best. With the temperature increasing, the conversion of naphthalene and selectivity of naphthol will rise., The conversion of naphthalene and selectivity of naphthol attained optimal value at $65 \mathrm{oC}$. With the reaction time increasing, the conversion of naphthalene and selectivity of naphthol were increased, and achieved little change at naphthalene conversion after 6h, After $8 \mathrm{~h}, 1$-naphthol's selectivity decreased, 2naphthol's selectivity kept smooth. With the increasing of hydrogen peroxide, the conversion of naphthalene and selectivity of naphthol increased. With the increasing amount of catalyst, the conversion of naphthalene and selectivity of naphthol were significantly increased.
\end{abstract}

\section{Introduction}

The formula of monohydric naphthols are $\mathrm{C}_{10} \mathrm{H}_{8} \mathrm{O}$, they are two isomers of 1-naphthol and 2naphthol. The molecular weight is 144.17. Naphthol is an important organic materials.,and mainly used in the production of synthetic fibers, synthetic rubber, plastics, pharmaceuticals, pesticides, perfumes, dyes and coatings, the market demand was increasing.

\section{Experimental section}

\subsection{Catalyst preparation}

The supported catalysts were prepared by impregnation of metal components.The specific preparation methods as follows: MCM-41 samples were calcined twice in a muffle furnace. Calcined $2 \mathrm{~h}$ at 250-350 oC, then kept at $350 \mathrm{oC}$ for 1-2 h more, calcined at $550 \mathrm{oC}$ for another 3-4h.And then Cooling down to room temperature for later use. The sample will changed from white to black, then to white during calcination. Metals (Fe, $\mathrm{Cu}, \mathrm{Mn}$ ) salt was dissolved in $25 \mathrm{~mL}$ methanol solution, and was stirred until completely dissolved, and then accurate weighing and adding MCM-41, stirring 4h, keeping 4h, rotary evaporation, and then dring overnight at $110 \mathrm{oC}$.After that,calcination for another $5 \mathrm{~h}$ at $550 \mathrm{oC}$,the Fe/MCM-41 catalyst was obtained.

\subsection{Characterization of the samples}

All catalysts were characterized byFourier transform infrared spectroscopy (FT-IR).

\subsection{Catalytic experiments}

$\mathrm{V}_{2} \mathrm{O}_{5}(15.8 \mathrm{~g})$ and $\mathrm{MoO}_{3}(0.91 \mathrm{~g})$ were dissolved in deionized water, then stirred and heated to $95^{\circ} \mathrm{C}\left( \pm 5^{\circ} \mathrm{C}\right)$ with stirring in oil bath. Driping the phosphoric acid solution $(1.15 \mathrm{~g}, 85 \mathrm{wt} \%)$ in above solution, and kept it under $95{ }^{\circ} \mathrm{C} \sim 100{ }^{\circ} \mathrm{C}$ for $18 \sim 24 \mathrm{~h}$ until the orange suspension became clear. Then the solution was filtered,drying under the $60^{\circ} \mathrm{C}$. We used liquid chromatography to test the sample. Liquid chromatography to Beijing LabTech Instruments Incorporated (LabTech LC600 HPLC), chromatography conditions: C18 column with a mobile phase of methanol/water $=58 / 42$, the detection wavelength to $220 \mathrm{~nm}$, flow rate at $0.8 \mathrm{~mL} / \mathrm{min}$. 


\section{Result and discussion}

\subsection{FT-IRresults}
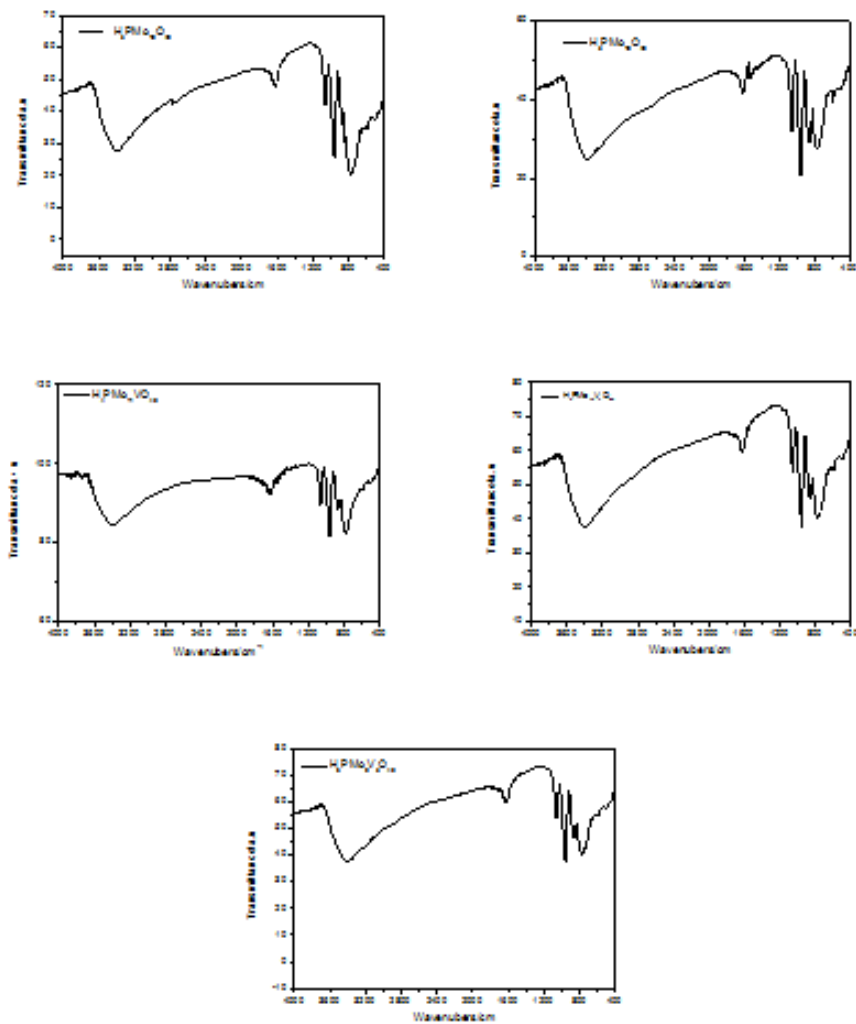

Figure1.FT-IR for Heteropolyacids

Figure 1. shows that it can be seen four Keggin structure peaks, the peak of the five heteropolyacids is between $700 \sim 1100 \mathrm{~cm}^{-1}$. Around $1000 \mathrm{~cm}^{-1}$ is PO's stretching vibration peak, around 900 $\mathrm{cm}^{-1}$ is Mo-O stretching vibration frequency bonds, $850 \mathrm{~cm}^{-1}$ ascribed to Mo-O-Mo bridges, the stretching vibration frequency peak around $750 \mathrm{~cm}-1$ attributed to the stretching vibration frequency of Mo-O-Mo bridge bond (oxygen bridge with groups $\mathrm{MoO}_{6}$ octahedral). Addition, $1620 \mathrm{~cm}^{-1}$ is close to the frequency of the bending vibration of water molecules in hydroxy.The analysis chart indicates that the compounds have Keggin structure of heteropoly acid.

\subsection{Catalytic activity}

Table 1. shows that all the heteropoly acid contain Keggin structure. The catalytic of $\mathrm{H}_{4} \mathrm{PMo}_{11} \mathrm{VO}_{40}$ is best, $\mathrm{H}_{3} \mathrm{PMo}_{12} \mathrm{O}_{40}$ and $\mathrm{H}_{3} \mathrm{PW}_{12} \mathrm{O}_{40}$ have a low yield of naphthol. The catalytic effect of the reaction production were that: $\mathrm{H}_{4} \mathrm{PMo}_{11} \mathrm{VO}_{40}>\mathrm{H}_{5} \mathrm{PW}_{10} \mathrm{~V}_{2} \mathrm{O}_{40}>\mathrm{H}_{6} \mathrm{PMo}_{9} \mathrm{~V}_{3} \mathrm{O}_{40}>$ $\mathrm{H}_{3} \mathrm{PW}_{12} \mathrm{O}_{40}>\mathrm{H}_{3} \mathrm{PMo}_{12} \mathrm{O}_{40}$. Obviously, the presence of vanadium in heteropolyacids increased the hydroxylation reaction in the conversion of naphthalene to naphthol. However, the $\mathrm{H}_{4} \mathrm{PMo}_{11} \mathrm{VO}_{40}$ property and the selectivity of naphthol was better than $\mathrm{H}_{5} \mathrm{PW}_{10} \mathrm{~V}_{2} \mathrm{O}_{40}$. Which was consistent with $\mathrm{Xu}$ Wenjun's report that the heteropolyacid stability affected catalytic activity, vanadium heteropoly acid catalysis are not as effective as a vanadium-substituted heteropolyacids may because that monoperoxovanadium active intermediate species in oxygen transfer reactions reduced because of its stability. 
Table 1. Miscellaneous impact of different types of acid hydroxylation of naphthalene naphthol

\begin{tabular}{cccc}
\hline Catalyst & $\begin{array}{c}\text { Naphtha- } \\
\text { leneCon.(\%) }\end{array}$ & \multicolumn{2}{c}{ Naphthol Sel. (\%) } \\
& & & \\
& 3.8 & 17.3 & 6.7 \\
\hline $\mathrm{H}_{3} \mathrm{PMo}_{12} \mathrm{O}_{40}$ & & & \\
& 4.3 & 29.2 & 9.8 \\
$\mathrm{H}_{3} \mathrm{PW}_{12} \mathrm{O}_{40}$ & 27.15 & 35.2 & 10.8 \\
$\mathrm{H}_{4} \mathrm{PMo}_{11} \mathrm{VO}_{40}$ & 29.47 & 26.6 & 9.4 \\
$\mathrm{H}_{5} \mathrm{PMo}_{10} \mathrm{~V}_{2} \mathrm{O}_{40}$ & 31.29 & 22.0 & 8.1 \\
$\mathrm{H}_{6} \mathrm{PMo}_{9} \mathrm{~V}_{3} \mathrm{O}_{40}$ & & & \\
\hline
\end{tabular}

Reaction conditions: Naphthalene (1000 mg, $7.81 \mathrm{mmol})$,catalyst(100mg), hydrogen peroxide (30 wt.\%, $2 \mathrm{~mL}, 19.7 \mathrm{mmol})$, acetonitrile $(25 \mathrm{~mL}), 65^{\circ} \mathrm{C}, 6 \mathrm{~h}$

\subsection{Reaction temperature}

Figure2. shows that with the increase of reaction temperature, the conversion of naphthalene and selectivity of naphthol increased at first. When the temperature was raised from $50{ }^{\circ} \mathrm{C}$ to $65^{\circ} \mathrm{C}$, naphthalene conversion rate increased by $8.3 \%$ to $27.15 \%$, the naphthol selectivity from $26.5 \%$ to $46 \%$; when the reaction temperature is $65^{\circ} \mathrm{C}$, the conversion of naphthalene reaches a maximum conversion rate of $27.15 \%$; when the catalyst does not exhibit high catalytic activity, the reaction temperature facilitated the reactive intermediates, accelerated the reaction rate, and improved the selectivity of naphthol. When the temperature rise to $70^{\circ} \mathrm{C}$, the selectivity of naphthol fell to $30 \%$, it is probably due to the temperature increasing the depth oxidation of naphthol and hydrogen peroxide decomposition. In addition, according to Arrhenius equation, at reaction temperature, the catalyst should exhibit higher catalytic activity, but $\mathrm{V}_{2} \mathrm{O}_{5}$ monomers can also be separated from the heteropolyacid skeleton. The generation naphthol have passivation effect of $\mathrm{VO}^{2+}$. With the temperature raised, the enhance activity of the catalyst does not compensate for the reduced activity of the catalyst $\mathrm{V}_{2} \mathrm{O}_{5}$ monomer removed. Further raising the reaction temperature, the selectivity of 1-naphthol in reducing, and elevated temperatures may be more accessible reduce. The boiling point of the acetonitrile which lead to naphthol concentration increases and in deep oxidation of 1-naphthol, 2naphthol. 2-naphthol's activity is weaker, the disadvantaged in the competition reaction impact may not obvious affect the selectivity. Therefore, the optimal reaction temperature is $65^{\circ} \mathrm{C}$, the catalyst showed higher conversion of naphthalene and selectivity of naphthol has reached $46 \%$.

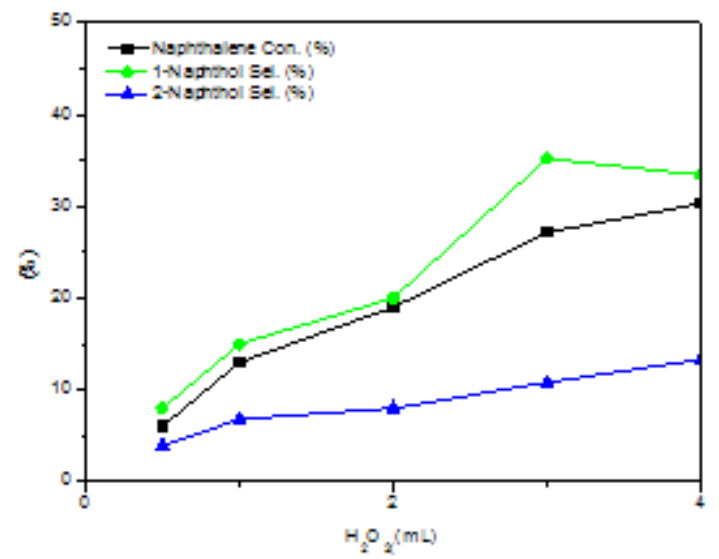

Figure2. Conversion-selectivity-reaction temperature in naphthalene hydroxylation;Reaction conditions: Naphthalene (1000 mg, $7.81 \mathrm{mmol}$ ), catalyst(100mg), hydrogen peroxide (30 wt.\%, $2 \mathrm{~mL}, 19.7 \mathrm{mmol}$ ), acetonitrile(25 mL), $8 \mathrm{~h}$ 


\subsection{Reaction time}

Figure3. shows that with the time increasing, the conversion of naphthalene and selectivity of naphthol have increasing trend. When the time reach $6 \mathrm{~h}$, the conversion trend is slower with the time; when reaction time was more than 6h, the selectivity of 1-naphthol decreased rapidly. Mainly with the high initial concentration of hydrogen peroxide reaction, naphthyl contacted with an oxidizing agent in the catalyst surface. With the reaction proceeds, the concentration of hydrogen peroxide have decreased. The probability of reactant molecules on the catalyst surface in contact have decrease. Resulting in the increasing of selectivity and conversion of naphthalene hydroxylation reaction slow. After more than 6h, naphthol was deep oxidation in the air, so the conversion rate decreased rapidly. Therefore, the best reaction time was $6 \mathrm{~h}$.

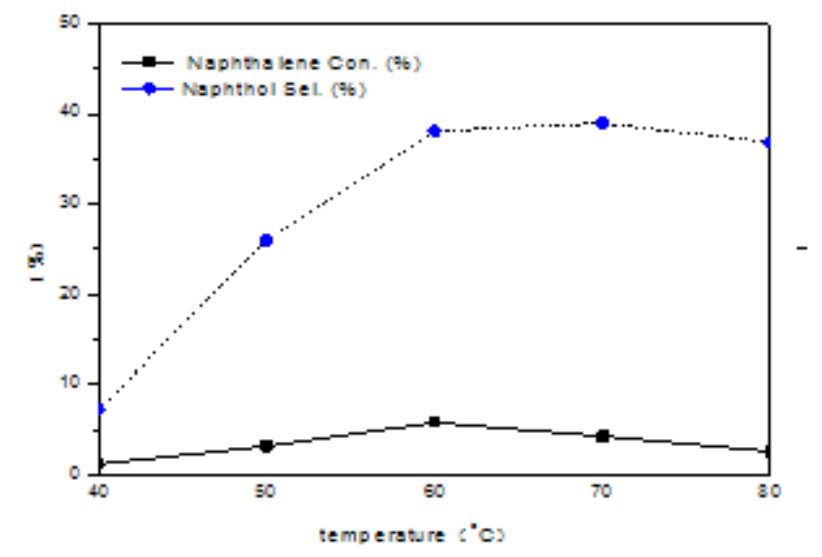

Figure 3. Conversion-selectivity-reaction temperature in naphthalene hydroxylation;Reaction conditions: Naphthalene (1000 mg, $7.81 \mathrm{mmol}$ ), catalyst(100mg),hydrogen peroxide (30 wt.\%, 2 mL, $19.7 \mathrm{mmol}$ ), acetonitrile $(25 \mathrm{~mL}), 65^{\circ} \mathrm{C}$.

\subsection{Reaction $\mathrm{H} 2 \mathrm{O} 2$}

Figure3. shows that with the increase of hydrogen peroxide, the conversion of naphthalene and selectivity of naphthol were increased. When the amount of hydrogen peroxide increased from $0.5 \mathrm{~mL}$ to $3.0 \mathrm{~mL}$, the conversion decreased from $6 \%$ to $27.15 \%$, the selectivity increased to $46 \%$ from $11.9 \%$. Mainly due to lower initial hydrogen peroxide concentration produced hydroxyl radicals and peroxide free a very small group, there is a certain induction period; with the increasing of hydrogen peroxide content, the free radicals generated can be efficiently and naphthalene molecules, accelerate the reaction of naphthol.However, with increasing hydrogen peroxide content 3.0 $\mathrm{mL}$ to $4.0 \mathrm{~mL}$, naphthalene conversion rate changed little. And 1-naphthol and 2-naphthol selectivity decreased slightly. Therefore, the appropriate amount of oxidant is $3.0 \mathrm{~mL}$.

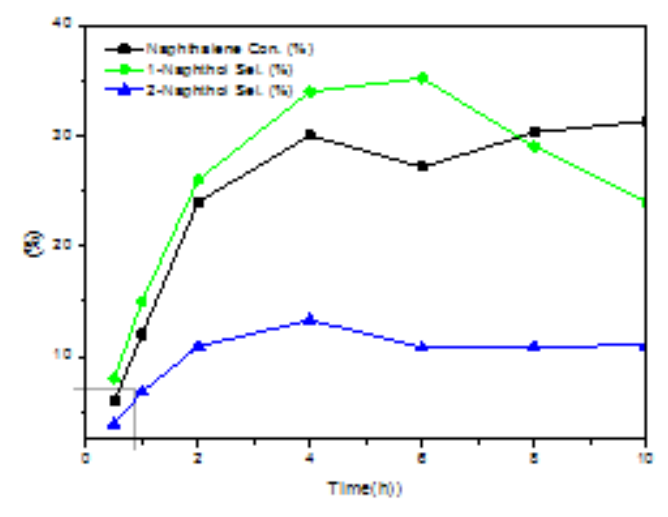

Figure 3. Conversion-selectivity-volume of $\mathrm{H}_{2} \mathrm{O}_{2}$ in naphthalene hydroxylation; Reaction conditions:Naphthalene (1000 mg, $7.81 \mathrm{mmol})$, catalyst(100mg), hydrogen peroxide (30 wt.\%), acetonitrile(25 $\mathrm{mL}), 65^{\circ} \mathrm{C}$. 


\section{Conclusion}

1. Prepared five heteropolyacid catalyst, the best reaction conditions: Naphthalene (1000 mg, $7.81 \mathrm{mmol})$, catalyst(100mg), hydrogen peroxide (30 wt.\%), acetonitrile(25mL), at $65^{\circ} \mathrm{C}$, under $\mathrm{H}_{4} \mathrm{PMo}_{11} \mathrm{VO}_{40}$ catalyst, the conversion of naphthalene was $30.3 \%$, selectivity of naphthol was $46.7 \%$.

2. Heteropolyacid catalysts have Keggin structure. The catalytic effect of $\mathrm{H}_{4} \mathrm{PMo}_{11} \mathrm{VO}_{40}$ is best, the selectivity of naphthol was 46.7\%, the catalytic effect as follows: $\mathrm{H}_{4} \mathrm{PMo}_{11} \mathrm{VO}_{40}>\mathrm{H}_{5} \mathrm{PW}_{10} \mathrm{~V}_{2} \mathrm{O}_{40}>$

$\mathrm{H}_{6} \mathrm{PMo}_{9} \mathrm{~V}_{3} \mathrm{O}_{40}>\mathrm{H}_{3} \mathrm{PW}_{12} \mathrm{O}_{40}>\mathrm{H}_{3} \mathrm{PMo}_{12} \mathrm{O}_{40}$.

3. With the temperature increases, the conversion of naphthalene and selectivity of naphthol will be increased. In close to $65^{\circ} \mathrm{C}$, naphthalene conversion rate was $27.15 \%$,reached the maximum, the naphthol selectivity was $46 \%$. With the increasing of reaction time, the conversion of naphthalene and selectivity of naphthol increased, and the naphthalene conversion had achieve little change after 6h. 1-naphthol selectivity decreased after 8h, and 2-naphthol selective retention kept smooth. With the increase of the content of hydrogen peroxide, the conversion of naphthalene and selectivity of naphthol increased. With the increasing of the amount of catalyst, the conversion of naphthalene and the selectivity of naphthol were significantly increased.

\section{References}

[1] Schulz T, Torborg C, Schäffner B, Huang J, Zapf A, Kadyrov R, et al. 2009. Practical imidazolebased phosphine ligands for selective palladium - catalyzed hydroxylation of aryl halides. Journal of Angewandte Chemie International Edition48: 918-921.

[2] Jänchen J, Stach H, Busio M, Wolput JHMCv. 1998. Microcalorimetric and spectroscopic studies of the acidic- and physisorption characteristics of MCM-41 and zeolites.Journal of Thermochimica Acta. 312: 33-45.

[3] Borraccino R, Kharoune M, Giot R, Agathos SN, Nyns E-J, Naveau HP, et al. 2001. Abiotic transformation of catechol and 1-naphthol in aqueous solution-Influence of environmental factors. Journal of Water Research35: 3729-3737.

[4] Yuan Shouqi et al. 2011. Numerical calculation of internal flow-induced noise in centrifugal pump volute. Journal of Drainage and Irrigation Machinery Engineering 3(29):93-98.

[5] Pretali L, Doria F, Verga D, Profumo A, Freccero M. Photoarylation/Alkylation of Bromonaphthols. 2009. The Journal of organic chemistry74: 1034-1041. 